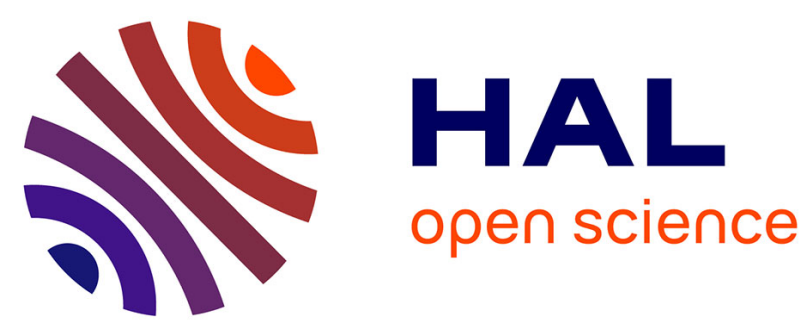

\title{
Non-uniformité de la distribution spatiale des états de surface dans le canal des transistors CMOS
}

B. Balland, C. Plossu, C. Choquet, V. Lubowiecki, J.L. Ledys

\section{To cite this version:}

B. Balland, C. Plossu, C. Choquet, V. Lubowiecki, J.L. Ledys. Non-uniformité de la distribution spatiale des états de surface dans le canal des transistors CMOS. Revue de Physique Appliquée, 1988, 23 (11), pp.1837-1845. 10.1051/rphysap:0198800230110183700 . jpa-00246012

\section{HAL Id: jpa-00246012 https://hal.science/jpa-00246012}

Submitted on 1 Jan 1988

HAL is a multi-disciplinary open access archive for the deposit and dissemination of scientific research documents, whether they are published or not. The documents may come from teaching and research institutions in France or abroad, or from public or private research centers.
L'archive ouverte pluridisciplinaire HAL, est destinée au dépôt et à la diffusion de documents scientifiques de niveau recherche, publiés ou non, émanant des établissements d'enseignement et de recherche français ou étrangers, des laboratoires publics ou privés. 


\title{
Non-uniformité de la distribution spatiale des états de surface dans le canal des transistors CMOS
}

\author{
B. Balland ( $\left.{ }^{1}\right)$, C. Plossu ( $\left.{ }^{(}\right)$, C. Choquet $\left({ }^{1}\right)$, V. Lubowiecki $\left({ }^{2}\right)$ et J. L. Ledys $\left({ }^{2}\right)$ \\ (1) Laboratoire de Physique de la Matière, Associé au Centre National de la Recherche Scientifique \\ (U.A. 358), Institut National des Sciences Appliquées, Bât. 502, 20, avenue Albert-Einstein, 69621 \\ Villeurbanne Cedex, France \\ (2) Matra-Harris Semiconducteurs, Centre électronique, La Chantrerie, Route de Gachet, 44075 Nantes \\ Cedex, France
}

(Reçu le 3 mars 1988, révisé le 2 mai 1988 et le 4 juillet 1988, accepté le 13 juillet 1988)

\begin{abstract}
Résumé. - Nous avons montré que les densités moyennes d'états d'interface obtenues par la technique de pompage de charge peuvent conduire à une surestimation du nombre réel de ces défauts en milieu de canal des transistors MOS, lorsque l'on suppose qu'ils sont uniformément distribués sur toute l'étendue de l'interface $\mathrm{Si}_{-} \mathrm{SiO}_{2}$. Les densités d'états de surface peuvent être plus élevées près de la source et du drain, même lorsque le transistor MOS n'a pas été soumis à une contrainte de vieillissement. Nous suggérons que cette augmentation est due aux dommages inévitablement produits au cours de certaines étapes du procédé d'élaboration des transistors et en particulier lors de la gravure plasma de l'électrode de grille. Les densités locales des états de surface doivent alors nécessairement être prises en compte, lors des études de vieillissement sur de tels composants. Nous proposons une nouvelle méthode de calcul permettant d'accéder, à partir des mesures en pompage de charge, à la densité d'états d'interface à proximité de la source et du drain avant et après injection de porteurs chauds.
\end{abstract}

\begin{abstract}
In this paper, we show that the interface state density in the middle of the channel obtained by charge pumping measurements on small size MOS transistors can be over-estimated, because of the actual non uniform spatial distribution along the $\mathrm{Si}_{-} \mathrm{SiO}_{2}$ interface. It appears to be necessary to take into account the local densities of surface states before degradation, when the charge pumping technique is used to analyse the rate of creation of new interface states in MOS transistors subjected to aging stresses. A new calculation method has been proposed which allows the determination of the surface states densities in the vicinity of the drain before and after channel hot carrier injection.
\end{abstract}

\section{Introduction.}

Les phénomènes de vieillissement des transistors MOS, par dégradation de l'interface $\mathrm{Si}_{-} \mathrm{SiO}_{2}$, sont actuellement très étudiés en raison de leur influence croissante avec la réduction des dimensions des composants MOS (transistors, mémoires EEPROM). La génération de nouveaux états d'interface et autres défauts électriquement actifs, initiée par effet d'un champ intense [1-2] et/ou par l'injection de porteurs chauds dans l'oxyde de grille joue un rôle primordial dans les phénomènes d'instabilités [3-4]. Pour étudier les mécanismes physiques de vieillissement, à l'origine de l'évolution des caractéristiques électriques des composants en technologie V.L.S.I., il est indispensable de disposer d'une technique de mesure de la densité des états de surface qui soit très performante (grandes sensibilité, précision et fiabilité).

Les mesures d'admittance, généralement utilisées pour l'étude précise relative à l'interface dans de simples capacités MOS, deviennent inexploitables dans le cas des transistors, dès que les capacités de la couche d'oxyde de grille deviennent inférieures à $10^{-12} \mathrm{~F}$. Il faut alors recourir à des techniques spécifiques telles que la méthode de l'inversion faible [5], la D.L.T.S. (deep level transient spectroscopy) [6], les mesures de bruit en $1 / f$ [7], ou le pompage de charge [8-11]. Cette dernière est la seule permettant de quantifier avec une bonne sensibilité (de l'ordre de $10^{9} \mathrm{~cm}^{-2} \cdot \mathrm{eV}^{-1}$ ) les densités d'états dans des transistors de petite géométrie. Le 
phénomène de pompage de charge a été décrit à l'origine par Brugler et al. [8]. Un modèle permettant d'accéder rapidement à la densité moyenne des états d'interface dans le canal, à leur section efficace de capture et à leur distribution énergétique dans la bande interdite du silicium a été proposé ultérieurement par Groeseneken et al. [11].

Il est généralement admis que les mécanismes à l'origine des instabilités électriques des composants MOS sont initiés par l'injection de porteurs chauds dans la couche d'oxyde de grille à partir de la région du canal située près du drain [12]. Il a été montré [12-16] que de nouveaux pièges sont alors créés à l'interface $\mathrm{Si}_{-} \mathrm{SiO}_{2}$, à proximité de la jonction drainsubstrat. La technique de pompage de charge a déjà été utilisée $[13,15,16]$ pour mettre en évidence cette dégradation non uniforme de l'interface $\mathrm{Si}-\mathrm{SiO}_{2}$. Maes et al. ont notamment proposé une méthode expérimentale de détermination de la distribution spatiale des états après une containte de vieillissement. Cette méthode repose sur l'hypothèse de base selon laquelle la répartition des défauts d'interface avant dégradation est uniforme le long du canal. Nous avons montré que cette hypothèse peut être erronée, et que la densité des états peut être plus élevée près de la source et du drain, compte tenu des conditions technologiques d'élaboration des transistors MOS. Nous proposons une méthode d'exploitation des mesures de pompage de charge permettant d'accéder, avant dégradation, aux densités locales des états d'interface, dont la connaissance s'avère ensuite indispensable pour une interprétation correcte des résultats de pompage de charge obtenus lors des études de vieillissement.

\section{Procédure expérimentale.}

2.1 ElABORATION DES TRANSISTORS MOS. - Les échantillons ont été élaborés selon un procédé de fabrication industriel de technologie CMOS 1,5 $\mu \mathrm{m}$. Les substrats silicium de nature cristalline $\mathrm{CZ}$ sont de type $\mathrm{P}$, de résistivité $5-8 \Omega . \mathrm{cm}$ et d'orientation cristallographique $\langle 100\rangle$. Les puits $\mathrm{N}^{-}$sont formés par une implantation de phosphore suivie d'une diffusion. La technique du Locos (« Local Oxidation on Silicon ») permet l'isolation interdispositif. Une implantation de champ préalable relève la concentration en additifs de dopage sous l'oxyde et donc le seuil des transistors parasites ; ces derniers sont ainsi mis dans l'état « off ».

Les tensions de seuil de deux transistors complémentaires NPN et PNP sont initialement différentes. Elles sont ajustées à une même valeur de $| \pm 0.75| \mathrm{V}$, par une implantation de bore à faible énergie dans les zones actives. A la fin du procédé, la concentration en surface, dans le canal, est : $2,6 \times 10^{16}$ atomes $/ \mathrm{cm}^{2}$ dans les zones $P$ et $8 \times$ $10^{15}$ atomes $/ \mathrm{cm}^{2}$ dans les zones $\mathrm{N}$.
Un procédé d'oxydation à $900^{\circ} \mathrm{C}$ conduit à un oxyde de grille de $300 \AA$, de bonne qualité électrique. Le matériau de grille est constitué par $5000 \AA$ de silicium polycristallin. Ce dernier est déposé sous basse pression soit entre 200 et $300 \mathrm{mT}$ à une température de $620^{\circ} \mathrm{C}$, par pyrolyse de silane $\mathrm{SiH}_{4}$. Il est ensuite dopé par diffusion de $\mathrm{POCl}_{3}$ à $950^{\circ} \mathrm{C}$; ce qui conduit à des résistances de couche de 20-25 $\Omega / \square$. L'électrode de grille est définie par gravure ionique réactive en chimie fluorée dans un réacteur monoplaque $\left(P=10^{-2} \mathrm{~Pa}\right)$. Les régions de source et de drain sont réalisées ultérieurement par implantation de $3 \times 10^{15}$ atomes d'arsenic $/ \mathrm{cm}^{2} \mathrm{du}$ côté $\mathrm{N}^{+}$et de $5 \times 10^{14}$ atomes de bore $/ \mathrm{cm}^{2}$ du côté $\mathrm{P}^{+}$. Une oxydation préalable (sur $800 \AA$ ) du silicium polycristallin permet d'éviter la canalisation des ions pendant l'implantation. Après dépôt d'une couche d'oxyde d'isolement entre métal et grille (7 $000 \AA ̊$ de $\mathrm{SiO}_{2}$ déposé en phase gazeuse par C.V.D.-atmosphérique et dopé phosphore), un recuit (à $1075^{\circ} \mathrm{C}$ pendant $8 \mathrm{mn}$ ) permet l'activation des implantations source/drain et le fluage de l'oxyde d'isolation. Après ouverture des contacts, $1000 \AA$ d'AlSi ( $1 \%$ de $\mathrm{Si}$ en poids) assure la métallisation du circuit. $\mathrm{Ce}$ film de métal est gravé sous plasma $\mathrm{Cl}_{2}+\mathrm{SiCl}_{4}$ pour former les interconnexions. Cette suite d'opérations se termine par un recuit à $450^{\circ} \mathrm{C}$ sous atmosphère réductrice $\left(\mathrm{N}_{2}+10 \% \mathrm{H}_{2}\right)$ pendant $30 \mathrm{mn}$; il a pour double effet de diminuer la résistivité du contact métal/silicium et de réduire les densités d'états de surface à l'interface $\mathrm{Si}-\mathrm{SiO}_{2}$.

Finalement la protection mécanique et la passivation sont assurées par une couche de $8000 \AA ̊$ d'oxyde dopé phosphore déposé en C.V.D.-atmosphérique.

2.2 TeChnique De POMPAge DE Charge. - Le montage expérimental que nous avons utilisé pour les mesures de pompage de charge est classique. Des impulsions de tension à profil triangulaire ou trapézoïdal sont appliquées sur la grille. Les jonctions drain-substrat et source-substrat sont identiquement polarisées en inverse.

Le modèle théorique proposé par Groeseneken et al. [11] montre que l'intensité $I_{\mathrm{Cp}}$ du courant de pompage dépend d'une part des caractéristiques du transistor (longueur effective $L_{\text {eff }}$ et largeur $W$ du canal, tension de seuil $V_{\mathrm{T}}$, tension de bandes plates $V_{\mathrm{FB}}$ ) et d'autre part des paramètres du signal impulsionnel de tension appliqué sur la grille (fréquence $f$, amplitude crête-crête $\Delta V_{\mathrm{G}}$, temps de montée $t_{\mathrm{r}}$ et temps de descente $t_{\mathrm{f}}$ ). En analysant les mécanismes d'émission à partir des états de surface, à l'aide du formalisme S.R.H. bien connu [17], on peut exprimer l'intensité $I_{\mathrm{CP}}$ à saturation du courant de pompage de charge, pour des signaux carrés ou en dent de scie, en fonction de la densité moyenne $N_{\text {it }}$ des états d'interface sur le domaine d'énergie exploré dans la bande interdite du silicium, et des 
sections efficaces de capture $\boldsymbol{\sigma}_{\mathrm{n}}$ et $\boldsymbol{\sigma}_{\mathrm{p}}$ pour les électrons et les trous respectivement, par [11] :

$$
\begin{array}{r}
I_{\mathrm{CP}}=2 q f W L_{\text {eff }} N_{\text {it }} k T\left\{\ln \left(v_{\text {th }} n_{\mathrm{i}} \sqrt{\sigma_{\mathrm{n}}} \boldsymbol{\sigma}_{\mathrm{p}}\right)+\right. \\
\left.+\ln \left({\sqrt{t_{\mathrm{r}}}}_{t_{\mathrm{f}}} \cdot\left|V_{\mathrm{FB}}-V_{\mathrm{T}}\right| /\left|\Delta V_{\mathrm{G}}\right|\right)\right\}
\end{array}
$$

avec $v_{\text {th }}$ la vitesse thermique des porteurs, et $n_{\mathrm{i}}$ la concentration intrinsèque dans le silicium.

Pour des impulsions à profil carré, on obtient une variation linéaire de $I_{\mathrm{CP}}$ avec la fréquence, comme le montre la figure 1. Dans le cas de signaux triangulaires, la quantité de charge émise et se recombinant à chaque cycle dépend de la fréquence, ce qui donne une variation plus complexe de $I_{\mathrm{CP}}$ avec $f$. Dans ce cas particulier, $t_{\mathrm{r}}$ et $t_{\mathrm{f}}$ s'expriment en fonction de la fréquence $f$ et du rapport $\alpha$ entre le temps de montée $t_{\mathrm{r}}$ et la période des pulses par :

$$
t_{\mathrm{r}}=\boldsymbol{\alpha} / f \text { et } \quad t_{\mathrm{f}}=\mathbf{1}-\boldsymbol{\alpha} / f .
$$

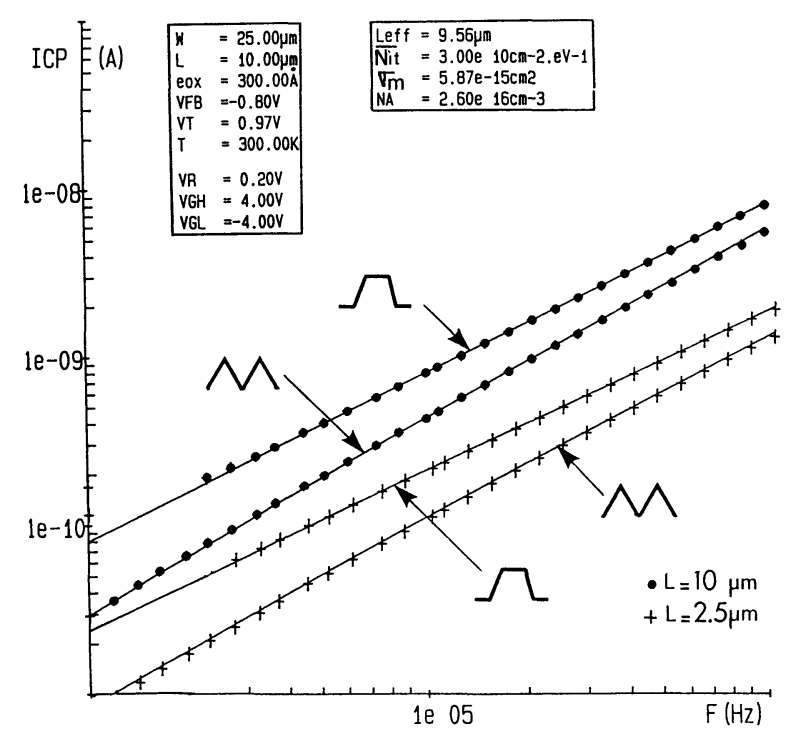

Fig. 1. - Courant de pompage de charge $I_{\mathrm{CP}}$ en fonction de la fréquence $f$ des pulses dans le cas de signaux carrés et triangulaires. Les points expérimentaux sont représentés par les symboles $(+)$ pour $L=2,5 \mu \mathrm{m}$ et $(\bullet)$ pour $L=10 \mu \mathrm{m}$. Les courbes théoriques $(-)$ sont obtenues à partir de l'expression (1) avec $N_{\mathrm{it}}=3 \times 10^{10} \mathrm{~cm}^{-2} \cdot \mathrm{eV}^{-1}$ pour $L=10 \mu \mathrm{m}, N_{\mathrm{it}}=4,4 \times 10^{10} \mathrm{~cm}^{-2} \cdot \mathrm{eV}^{-1}$ pour $L=$ $2,5 \mu \mathrm{m}$ et $\sigma_{\mathrm{m}}=5,9 \times 10^{-15} \mathrm{~cm}^{2}$.

[Frequency dependence of the charge pumping current for square and triangular pulses. The symbols are measured data $(+)$ for $L=2.5 \mu \mathrm{m}$, ( $\bullet)$ for $L=10 \mu \mathrm{m}$, while the solid lines are calculated using equation (1) $\left(N_{\mathrm{it}}=3 \times\right.$ $10^{10} \mathrm{~cm}^{-2} \cdot \mathrm{eV}^{-1} \quad$ for $L=10 \mu \mathrm{m}, \quad N_{\mathrm{it}}=4,4 \times$ $10^{10} \mathrm{~cm}^{-2} \mathrm{eV}^{-1}$ for $\left.L=2.5 \mu \mathrm{m}, \sigma_{\mathrm{m}}=5.9 \times 10^{-15} \mathrm{~cm}^{2}\right)$.]

Conformément à l'expression précédente, si $\alpha$ est maintenu constant, le rapport $I_{\mathrm{CP}} / f$ varie linéairement avec $\log f$. La détermination de la pente $p$ de la courbe $I_{\mathrm{CP}} / f(\log f)$ et son extrapolation à
$I_{\mathrm{CP}} / f=0$ (courant nul, pour une fréquence $f_{0}$ ), conduit aisément à la densité moyenne $N_{\text {it }}$ des états d'interface et à la valeur moyenne géométrique des sections efficaces de capture pour les électrons et les trous $\left(\sigma_{\mathrm{m}}=\sqrt{\boldsymbol{\sigma}_{\mathrm{n}}}, \boldsymbol{\sigma}_{\mathrm{p}}\right)$ :

$$
\begin{aligned}
& N_{\text {it }}=\frac{\log e}{2 q W L_{\text {eff }} k T} \cdot \frac{\mathrm{d}\left(I_{\mathrm{CP}} / f\right)}{\mathrm{d} \log f} \\
& \boldsymbol{\sigma}_{\mathrm{m}}=\sqrt{\boldsymbol{\sigma}_{\mathrm{n}}} \boldsymbol{\sigma}_{\mathrm{p}}=\frac{1}{v_{\text {th }} n_{\mathrm{i}}} \cdot \frac{\left|\Delta V_{\mathrm{G}}\right|}{\left|V_{\mathrm{FB}}-V_{\mathrm{T}}\right|} \times \\
& \quad \times \frac{f_{0}}{\left\{\boldsymbol{\alpha}(1-\boldsymbol{\alpha})^{1 / 2}\right\}} .
\end{aligned}
$$

Les courbes expérimentales $I_{\mathrm{CP}}(f)$ pour $L=$ $2,5 \mu \mathrm{m}$ et $L=10 \mu \mathrm{m}$ sont comparées sur la figure 1 aux courbes théoriques obtenues à l'aide de l'expression (1) avec $N_{\text {it }}$ et $\sigma_{\mathrm{m}}$ déterminées par la méthode décrite ci-dessus. Le bon accord obtenu montre la validité du modèle, tout au moins en ce qui concerne l'influence de la fréquence des pulses. On peut alors adopter une procédure expérimentale très rapide pour la détermination de $N_{\text {it }}$ et $\sigma_{\mathrm{m}}$, qui nécessite simplement deux mesures de l'intensité du courant $I_{\mathrm{CP}}$, l'une pour des pulses triangulaires, l'autre pour des pulses trapézoïdaux de même fréquence et de même amplitude. A partir de l'expression théorique (1) de l'intensité, on calcule aisément les valeurs numériques de $N_{\text {it }}$ et $\boldsymbol{\sigma}_{\mathrm{m}}$.

\section{Distribution spatiale des états d'interface.}

Contrairement aux résultats expérimentaux obtenus par Groeseneken et al. [11], la variation du courant de pompage de charge $I_{\mathrm{CP}}$ avec la tension de polarisation inverse $V_{\mathrm{R}}$ des jonctions, dans le cas des échantillons étudiés ici, n'est pas conforme à l'expression (1), si l'on considère une distribution uniforme des états le long du canal. Nous avons donc été amenés à envisager une répartition spatiale non uniforme des défauts d'interface avant toute contrainte de vieillissement.

Il a déjà été montré $[13,15,16]$ que la technique de pompage de charge permet d'évaluer la répartition spatiale des états sur des échantillons ayant subi des contraintes de vieillissement, par comparaison des courants de substrat mesurés avant et après dégradation. Dans la référence [13], les auteurs font l'hypothèse selon laquelle la surface dégradée du canal s'étend sur $0.1 \mu \mathrm{m}$. Dans cette région la densité d'états d'interface moyenne passe de $1,7 \times$ $10^{10} \mathrm{~cm}^{-2} \cdot \mathrm{eV}^{-1}$ à $5 \times 10^{11} \mathrm{~cm}^{-2} \cdot \mathrm{eV}^{-1}$. Ces résultats sont obtenus par la méthode de pompage de charge mais la procédure expérimentale suivie n'est pas explicitée. Dans la référence [15], aucune mesure quantitative de la densité d'états n'est donnée. Seule l'observation qualitative des courbes de $I_{\mathrm{CP}}$ en fonction du niveau bas des pulses avant et après 
contrainte est exploitée. Dans la référence [16], les auteurs proposent une méthode de détermination de la distribution spatiale des états d'interface dans le canal. Leur démarche est différente de la nôtre. Ils attribuent la diminution du courant de pompage de charge avec la tension inverse de drain et source, sur des échantillons non contraints, aux seules variations de la longueur effective du canal et de la tension de seuil du transistor, en supposant que la densité initiale des états est constante le long du canal. A partir des mesures expérimentales de $I_{\mathrm{CP}}$ en fonction de $V_{\mathrm{R}}$ et de la variation théorique de $L_{\text {eff }}$ et $V_{\mathrm{T}}$ avec $V_{\mathrm{R}}$, ils en déduisent le dopage effectif $N_{\text {B }}$ du substrat. Par comparaison des courants de pompage de charge avant et après dégradation, ils obtiennent ensuite la distribution spatiale des états après vieillissement. Cette démarche n'a pu être adoptée dans le cas des échantillons étudiés ici. En effet une valeur incohérente du dopage aurait été nécessaire pour rendre compte de la diminution du courant $I_{\mathrm{CP}}$ avec $V_{\mathrm{R}}$. Nous avons donc été amenés à envisager une diminution de la densité d'états lorsque l'on s'éloigne des bords du canal. La méthode décrite ici est donc différente de celle proposée dans la référence [16] puisqu'elle s'applique à des échantillons non contraints. Dans le paragraphe 4, nous intégrons les densités locales des états avant dégradation dans l'exploitation des mesures de pompage de charge après vieillissement des transistors.

3.1 RÉSUltats EXPÉRIMENTAUX. - La distribution spatiale des états d'interface le long du canal est obtenue en utilisant la modulation de la longueur effective $L_{\text {eff }}$ du canal par la tension inverse $V_{\mathrm{R}}$ des jonctions source/substrat et drain/substrat. Lorsque $L_{\text {eff }}$ diminue, les états d'interface situés dans les régions désertées aux deux extrémités du canal (dans les régions de source et de drain) ne contribuent plus au courant de pompage de charge. De plus la tension de seuil $V_{\mathrm{T}}$ du transistor augmente avec $V_{\mathrm{R}}$. D'après l'expression (1), le courant de substrat $I_{\mathrm{CP}}$ diminue.

Les caractéristiques expérimentales $I_{\mathrm{CP}}\left(V_{\mathrm{GL}}\right)$ pour $L=2,5 \mu \mathrm{m}$ sont représentées sur la figure $2 \mathrm{en}$ fonction de $V_{\mathrm{R}}$. On observe effectivement un décalage des tensions qui correspondent au seuil d'apparition de $I_{\mathrm{CP}}$, dû à l'augmentation de $V_{\mathrm{T}}$, et également une diminution de la valeur de saturation du courant.

Les variations de $L_{\text {eff }}$ et $V_{\mathrm{T}}$ avec $V_{\mathrm{R}}$ ont été modélisées $[16,17]$, en prenant en compte la variation de la largeur $W_{\mathrm{m}}$ des régions désertées autour des jonctions drain-substrat et source-substrat. $\mathrm{La}$ tension de diffusion des jonctions étant prise en compte dans $V_{\mathrm{R}}$ qui est donc considérée comme la tension de jonction, $W_{\mathrm{m}}$ est donnée par:

$$
W_{\mathrm{m}}\left(V_{\mathrm{R}}\right)=\left\{\frac{2 \varepsilon_{\mathrm{si}}}{q N_{\mathrm{A}}}\left(V_{\mathrm{R}}+2 \Psi_{\mathrm{B}}\right)\right\}^{1 / 2}
$$

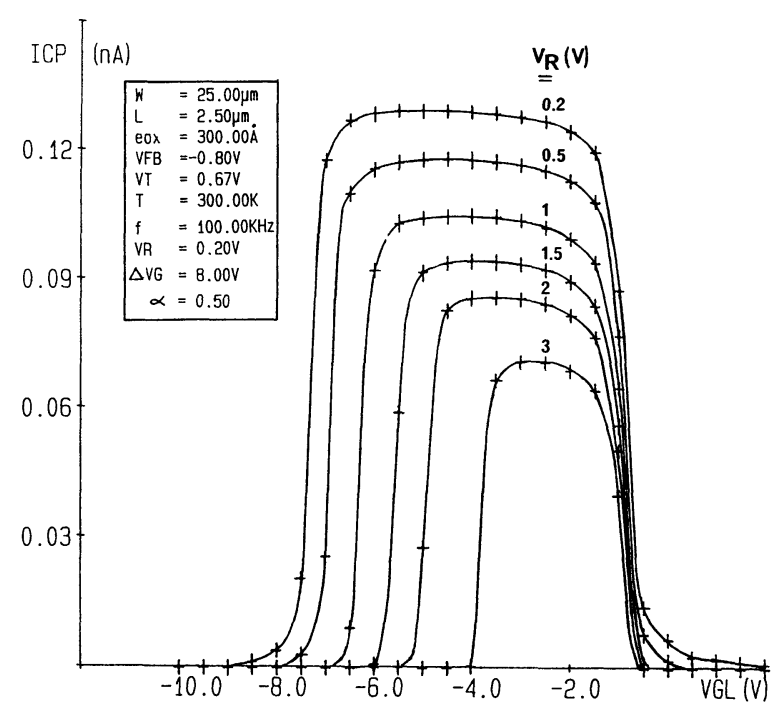

Fig. 2. - Variations du courant $I_{\mathrm{CP}}$ de pompage de charge avec le niveau bas $V_{\mathrm{GL}}$ des pulses, en fonction de la tension de polarisation inverse $V_{\mathrm{R}}$ de source et de drain.

[Charge pumping current $I_{\mathrm{CP}}$ as a function of gate pulse base level $V_{\mathrm{GL}}$ with the reverse bias voltage $V_{\mathrm{R}}$ applied to the source and drain as a parameter.]

$L$ étant la longueur du canal, sa longueur effective s'exprime alors en fonction de $V_{\mathrm{R}}$ par :

$$
L_{\text {eff }}\left(V_{\mathrm{R}}\right)=L-2 W_{\mathrm{m}}\left(V_{\mathrm{R}}\right) .
$$

La tension de seuil est obtenue à l'aide de l'expression ci-dessous [17]:

$$
\begin{aligned}
V_{\mathrm{T}}=V_{\mathrm{FB}} & +2 \Psi_{\mathrm{B}}+V_{\mathrm{R}}+ \\
& +\frac{\left\{2 q \varepsilon_{\mathrm{si}} N_{\mathrm{A}}\left(V_{\mathrm{R}}+2 \Psi_{\mathrm{B}}\right)\right\}^{1 / 2}}{\varepsilon_{\mathrm{ox}} / e_{\mathrm{ox}}}+\Delta V_{\mathrm{T}}
\end{aligned}
$$

où

$$
\Delta V_{\mathrm{T}}=-\frac{q N_{\mathrm{A}} W_{\mathrm{m}} r_{\mathrm{j}}}{L \cdot \varepsilon_{\mathrm{ox}} / e_{\mathrm{ox}}}\left\{\left[1+2 W_{\mathrm{m}} / r_{\mathrm{j}}\right]^{1 / 2}-1\right\}
$$

$N_{\mathrm{A}}$ est le dopage en surface, $r_{\mathrm{j}}$ la profondeur des jonctions, $q \Psi_{\mathrm{B}}$ la différence d'énergie entre le niveau de Fermi et le niveau intrinsèque dans le silicium, $e_{\mathrm{ox}}$ l'épaisseur de la couche d'oxyde de grille, $\varepsilon_{\mathrm{si}}$ et $\varepsilon_{\mathrm{ox}}$ les permittivités diélectriques de $\mathrm{Si}$ et . $\mathrm{SiO}_{2}$ respectivement.

Les variations de $I_{\mathrm{CP}}$ avec $V_{\mathrm{R}}$ ont été reproduites sur la figure 3 pour des signaux triangulaires et trapézoïdaux. Les courbes théoriques sont obtenues à l'aide de l'expression (1), en supposant une répartition uniforme des états d'interface le long du canal $N_{\text {it }}(x)=N_{\text {it }}$, valeur déterminée pour un faible $V_{\mathrm{R}}$. On observe que le courant $I_{\mathrm{CP}}$ diminue plus rapidement que ne le prévoit le modèle. Cette diminution ne peut donc pas s'expliquer par la seule variation de $L_{\text {eff }}$ et $V_{\mathrm{T}}$ avec $V_{\mathrm{R}}$. Il est nécessaire d'envisager une diminution de $N_{\text {it }}$ si la distance par 


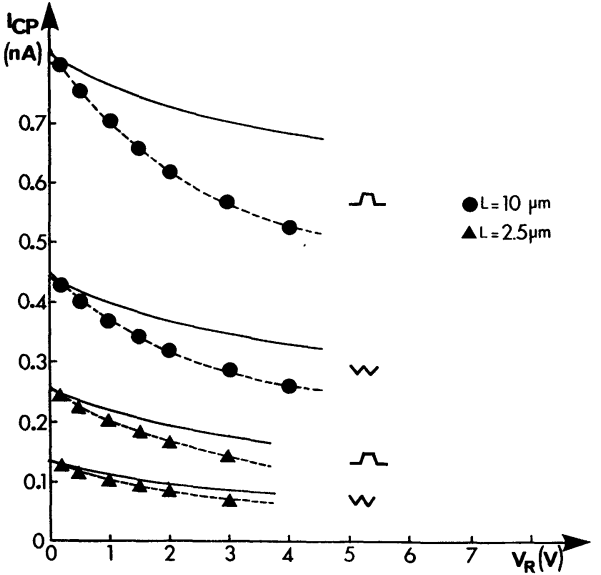

Fig. 3. - Variations du courant de pompage de charge avec $V_{\mathrm{R}}$, dans le cas de pulses carrés et triangulaires. Les points expérimentaux sont représentés par les symboles pour $L=2,5 \mu \mathrm{m}$ et $L=10 \mu \mathrm{m}$. Les courbes théoriques sont calculées en supposant une distribution uniforme des états d'interface le long du canal.

$\left[V_{\mathrm{R}}\right.$ dependence of charge pumping current for square and triangular pulses. The symbols are measured data for $L=2.5 \mu \mathrm{m}$ and $L=10 \mu \mathrm{m}$, while the solid lines are calculated curves assuming a uniform distribution of the surface states.]

rapport aux extrémités du canal augmente. Nous avons développé une méthode de calcul permettant d'obtenir les valeurs de $N_{\text {it }}(x)$ près de la source et du drain, à partir des valeurs expérimentales de $N_{\text {it }}\left(L_{\text {eff }}\right)$.

Si l'on prend l'origine des $x$ au milieu du canal, et en considérant que la distribution énergétique reste la même le long du canal, $N_{\text {it }}\left(L_{\text {eff }}\right)$ s'exprime par :

$$
N_{\text {it }}\left(L_{\text {eff }}\right)=\frac{1}{L_{\text {eff }}} \cdot \int_{-L_{\text {eff }} / 2}^{L_{\text {eff }} / 2} N_{\text {it }}(x) \mathrm{d} x .
$$

En dérivant l'expression ci-dessus par rapport à $L_{\text {eff }} / 2$, on obtient :

$$
\begin{aligned}
N_{\text {it }}\left(L_{\text {eff }} / 2\right)+ & N_{\text {it }}\left(-L_{\text {eff }} / 2\right)= \\
& =2 L_{\text {eff }} \frac{\mathrm{d} N_{\text {it }}\left(L_{\text {eff }}\right)}{\mathrm{d} L_{\text {eff }}}+2 N_{\text {it }}\left(L_{\text {eff }}\right) .
\end{aligned}
$$

En supposant $\quad N_{\text {it }}\left(L_{\text {eff }} / 2\right)=N_{\text {it }}\left(-L_{\text {eff }} / 2\right)$, hypothèse valable uniquement pour un transistor non dégradé, n'ayant subi aucune contrainte de vieillissement, il vient :

$$
\begin{aligned}
N_{\text {it }}\left(L_{\text {eff }} / 2\right) & =N_{\text {it }}\left(-L_{\text {eff }} / 2\right) \\
& =L_{\text {eff }} \frac{\mathrm{d} N_{\text {it }}\left(L_{\text {eff }}\right)}{\mathrm{d} L_{\text {eff }}}+N_{\text {it }}\left(L_{\text {eff }}\right) .
\end{aligned}
$$

Il est important de noter que la technique de pompage de charge permet de déterminer les valeurs moyennes $N_{\text {it }}\left(L_{\text {eff }}\right)$ avec une bonne précision, de l'ordre de $5 \%$. Il est donc correct d'utiliser ces valeurs pour le calcul des densités locales à partir de l'équation (10).

Les mesures expérimentales de $I_{\mathrm{CP}}$ en fonction de $V_{\mathrm{R}}$ permettent de déterminer à l'aide des expressions $(1,4$ et 5$)$, les variations de $N_{\text {it }}$ avec $\left(L-L_{\text {eff }}\right) / 2$. La figure 4 montre que les valeurs obtenues pour des signaux triangulaires et trapézoïdaux concordent parfaitement entre elles. La répartition spatiale $N_{\text {it }}(x)$ des états d'interface est alors déterminée à l'aide de l'expression (10). On observe sur la figure 5 une forte augmentation de $N_{\text {it }}$ près de la source et du drain. $N_{\text {it }}$ atteint $2 \times 10^{11} \mathrm{~cm}^{-2} \mathrm{eV}^{-1}$ aux extrémités du canal. Connaissant la densité moyenne $N_{\text {it }}$ sur toute la longueur du canal $\left(N_{\text {it }}=3 \times\right.$

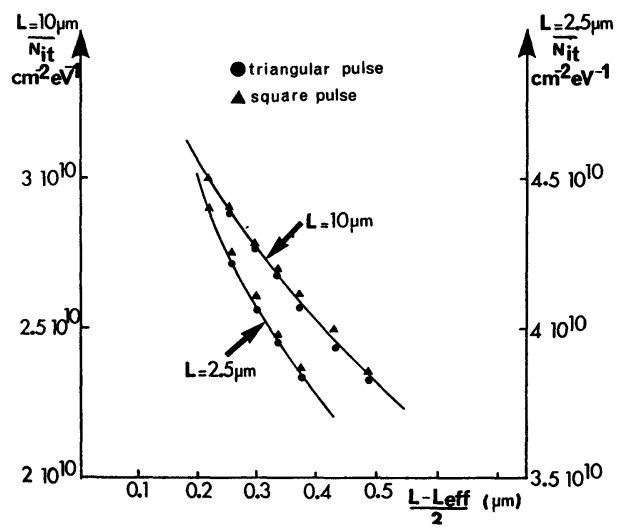

Fig. 4. - Variations de la valeur moyenne $N_{\text {it }}$ de la densité des états d'interface avec $\left(L-L_{\text {eff }}\right) / 2$ pour des pulses carrés et triangulaires.

[Experimental decrease of the $N_{\text {it }}$ mean value of interface state density with $\left(L-L_{\text {eff }}\right) / 2$ for both triangular and square pulses.]

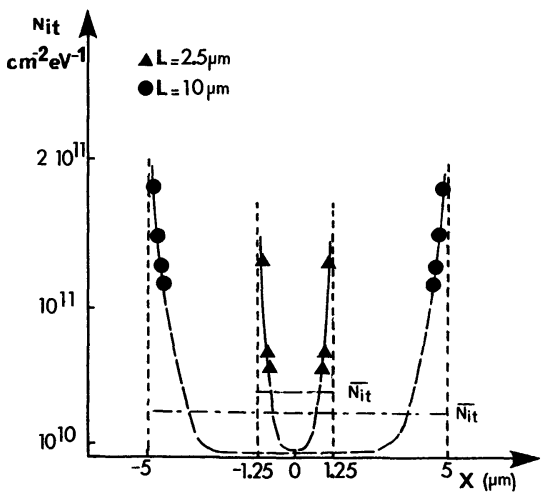

Fig. 5. - Distribution spatiale des états d'interface à proximité de la source et du drain pour $L=2,5 \mu \mathrm{m}$ et $L=10 \mu \mathrm{m}$.

[Spatial distribution of surface states in the vicinity of source and drain junctions for $L=2.5 \mu \mathrm{m}$ and $L=$ $10 \mu \mathrm{m}$. 
$10^{10} \mathrm{~cm}^{-2} \mathrm{eV}^{-1}$ pour $L=10 \mu \mathrm{m}$ et $N_{\mathrm{it}}=4,4 \times$ $10^{10} \mathrm{~cm}^{-2} \mathrm{eV}^{-1}$ pour $\left.L=2,5 \mu \mathrm{m}\right)$, on peut en déduire que la densité d'états d'interface en milieu de canal est inférieure à $10^{10} \mathrm{~cm}^{-2} \mathrm{eV}^{-1}$.

3.2 Discussion. - Contrairement aux résultats obtenus par d'autres $[11,16]$, les variations du courant de pompage de charge avec la tension inverse de source et drain ne sont pas conformes au modèle théorique, si on considère une distribution spatiale uniforme le long du canal. Nous avons montré que les résultats expérimentaux obtenus peuvent s'expliquer par une densité d'états d'interface plus élevée près de la source et du drain qu'en milieu de canal. Cette augmentation pourrait être associée à la présence d'impuretés chimiques, introduites au cours d'une ou plusieurs phases du procédé d'élaboration des transistors. Plus particulièrement, il a été établi $[19,20]$ que l'étape de définition des électrodes de grille par gravure ionique réactive du silicium polycristallin pourrait être une des plus critiques en ce qui concerne la dégradation de l'interface $\mathrm{Si}-\mathrm{SiO}_{2}$. Cependant, cette technique de gravure sous plasma a progressivement remplacé les méthodes d'attaque chimique ; sa grande anisotro-" pie, sa bonne sélectivité et sa reproductibilité lui ont permis de s'imposer en technologie V.L.S.I. qui nécessite un bon contrôle dimensionnel des motifs submicroniques. Mais de par son principe même, elle nécessite un bombardement des surfaces à graver par des ions de grande énergie créant des dommages dans les couches sous-jacentes [20]. De plus une contamination en fin de gravure par les espèces chimiques excitées lors de la décharge ou par les composés issus des réactions chimiques avec la couche gravée peut se produire. Elle se traduit alors par une dégradation volumique de la couche d'oxyde de grille et une augmentation non uniforme de la densité d'états d'interface, plus accentuée près de la source et du drain. Il en résulte une dérive de la tension de seuil et de la transconductance du canal des transistors.

L'étape ultérieure de formation des régions source et drain par implantation ionique, suivie d'un recuit à haute température, pour activation des implants, peut également favoriser la diffusion des impuretés contaminantes (fluor, carbone, fer, nickel, ...) introduites lors des étapes de gravure par plasma ou d'implantation ionique $[18,19]$.

\section{Distribution spatiale des états après dégradation : méthode de calcul.}

Lorsque les états d'interface ne sont pas uniformément distribués avant toute contrainte de vieillissement, les densités locales des états doivent nécessairement être prises en compte lors de l'exploitation des mesures de pompage de charge sur un échantil- lon contraint. Pour évaluer les variations de la densité d'états d'interface à proximité du drain, après une injection de porteurs chauds à partir du canal, le courant de pompage de charge doit être mesuré avant et après dégradation dans les mêmes conditions de polarisation de la grille et pour un même potentiel inverse $V_{\mathrm{R}}$ appliqué à la source et au drain.

L'intensité du courant de pompage de charge pour un échantillon n'ayant subi aucune contrainte est donnée par :

$$
I_{\mathrm{CP} 1}=K_{1} \cdot \int_{-L_{\mathrm{eff}} / 2}^{L_{\mathrm{eff}} / 2} N_{\mathrm{it} 1}(x) \mathrm{d} x
$$

où $K_{1}$ est une constante obtenue à partir de l'équation (1) :

$$
\begin{aligned}
K_{1}= & 2 q f W k T L_{\mathrm{eff}}\left[\ln \left(v_{\mathrm{th}} n_{\mathrm{i}} \sqrt{\boldsymbol{\sigma}_{\mathrm{n} 1}} \sigma_{\mathrm{p} 1}\right)+\right. \\
& \left.+\ln \left(\sqrt{t_{r}} t_{\mathrm{f}} \cdot\left|V_{\mathrm{FB} 1}-V_{\mathrm{T} 1}\right| /\left|\Delta V_{\mathrm{G}}\right|\right)\right] .
\end{aligned}
$$

D'autre part, le courant de pompage de charge mesuré après contrainte peut s'écrire :

$$
I_{\mathrm{CP} 2}=K_{2} \cdot \int_{-L_{\text {eff }} / 2}^{L_{\mathrm{eff}} / 2} N_{\mathrm{it} 2}(x) \mathrm{d} x
$$

avec

$$
\begin{array}{rl}
K_{2}=2 q & f W k T L_{\mathrm{eff}}\left[\ln \left(v_{\mathrm{th}} n_{\mathrm{i}} \sqrt{\boldsymbol{\sigma}_{\mathrm{n} 2}} \boldsymbol{\sigma}_{\mathrm{p} 2}\right)+\right. \\
& \left.+\ln \left(\sqrt{t_{\mathrm{r}}} t_{\mathrm{f}} \cdot\left|V_{\mathrm{FB} 2}-V_{\mathrm{T} 2}\right| /\left|\Delta V_{\mathrm{G}}\right|\right)\right]
\end{array}
$$

$\sigma_{\mathrm{n} 1}$ et $\sigma_{\mathrm{p} 1}$ sont les sections efficaces de capture des états d'interface préexistants tandis que $\sigma_{\mathrm{n} 2}$ et $\sigma_{\mathrm{p} 2}$ sont les sections efficaces de capture des états d'interface après dégradation.

Les variations de la tension de bandes plates et de la tension de seuil durant les contraintes sont dues à un éventuel piégeage de porteurs de charge dans le volume de la couche d'oxyde de grille. On supposera ici que les profondeurs des zones désertées autour de la source et du drain sont les mêmes que sur un échantillon non contraint ; cette hypothèse n'est correcte que pour une dégradation modérée, si l'augmentation de $N_{\text {it }}$ à proximité du drain n'est pas trop importante.

En prenant la dérivée du rapport $U=I_{\mathrm{CP} 2} / I_{\mathrm{CP} 1}$ par rapport à $V_{\mathrm{R}}$ et en supposant que la densité d'états d'interface près de la source n'est pas modifiée hypothèse couramment admise, c'est-à-dire :

$$
N_{\text {it } 1}\left(-L_{\text {eff }} / 2\right)=N_{\text {it } 1}\left(L_{\text {eff }} / 2\right)=N_{\text {it } 2}\left(-L_{\text {eff }} / 2\right)
$$

on obtient :

$$
\begin{aligned}
& \frac{\mathrm{d} U}{\mathrm{~d} V_{\mathrm{R}}}=\frac{K_{1}}{K_{2}} \cdot U \cdot \frac{\mathrm{d}\left(K_{2} / K_{1}\right)}{\mathrm{d} V_{\mathrm{R}}}+K_{2} \frac{\mathrm{d}\left(L_{\mathrm{eff}} / \mathrm{d} V_{\mathrm{R}}\right)}{2 I_{\mathrm{CP} 1}} \times \\
& \quad \times\left[N_{\mathrm{it} 2}\left(L_{\mathrm{eff}} / 2\right)-\left(1-\frac{2 K_{1}}{K_{2}} U\right) \cdot N_{\mathrm{it} 1}\left(L_{\mathrm{eff}} / 2\right)\right] .
\end{aligned}
$$


La densité d'états de surface près du drain après dégradation est donnée finalement par :

$$
\begin{aligned}
& N_{\mathrm{it} 2}\left(L_{\mathrm{eff}} / 2\right)=\frac{2 I_{\mathrm{CP} 1}}{K_{2}\left(\mathrm{~d} L_{\mathrm{eff}} / \mathrm{d} V_{\mathrm{R}}\right)}\left[\frac{\mathrm{d} U}{\mathrm{~d} V_{\mathrm{R}}}-\frac{K_{1}}{K_{2}} \times\right. \\
& \left.\times U \cdot \frac{\mathrm{d}\left(K_{2} / K_{1}\right)}{\mathrm{d} V_{\mathrm{R}}}\right]+\left(\frac{2 K_{1}}{K_{2}} \cdot U-1\right) \cdot N_{\mathrm{it} 1}\left(L_{\mathrm{eff}} / 2\right)
\end{aligned}
$$

$U$ et $\mathrm{d} U / \mathrm{d} V_{\mathrm{R}}$ sont obtenus à partir des mesures expérimentales de $I_{\mathrm{CP} 1}$ et $I_{\mathrm{CP} 2}$ en fonction de $V_{\mathrm{R}} \cdot \mathrm{d} L_{\text {eff }} / \mathrm{d} V_{\mathrm{R}}$ et $\mathrm{d}\left(K_{2} / K_{1}\right) / \mathrm{d} V_{\mathrm{R}}$ peuvent être déterminés à partir des équations $(4,5,12$ et 14$)$. Les développements du calcul sont donnés en annexe. D'après l'équation (16), la distribution spatiale des états de surface générés près du drain, lors des contraintes de vieillissement peut être déterminée (les résultats seront publiés ultérieurement).

\section{Conclusion.}

La technique de pompage de charge est une méthode d'investigation des états d'interface offrant à la fois une bonne sensibilité d'utiliser des transistors de technologie V.L.S.I. Elle permet une détermination très rapide et très précise de la densité moyenne des états d'interface dans le canal. Sa facilité de mise en œuvre avec informatisation, lui permettrait d'être aisément intégrée dans une filière de contrôle de procédés de fabrication en milieu industriel.

Cependant, pour une étude plus précise de l'interface $\mathrm{Si}-\mathrm{SiO}_{2}$, le modèle d'exploitation doit être affiné. Nous avons montré que la technique de pompage de charge permet d'évaluer la répartition spatiale des états à l'interface $\mathrm{Si}-\mathrm{SiO}_{2}$ sur des transistors natifs. Il est en effet apparu que cette répartition peut ne pas être uniforme le long du canal. Notamment nous avons observé une augmentation de la densité d'états à proximité des jonctions drain-substrat et source-substrat. Les valeurs moyennes de $N_{\text {it }}$ fournies par la technique de pompage de charge surestiment alors la densité réelle d'états en milieu de canal. Lors des études de vieillissement sur les transistors MOS, les densités locales des états de surface avant dégradation doivent alors être nécessairement intégrées dans les calculs d'exploitation des mesures de pompage de charge effectuées sur des composants soumis à des contraintes de vieillissement.

La technique de pompage de charge constitue donc un outil d'analyse précieux, dans le cadre d'une étude plus approfondie des dégradations introduites par certaines étapes technologiques de fabrication des circuits, comme par exemple les étapes de gravure par plasma, ou par des contraintes électriques, optiques ou thermiques.

\section{Annexe.}

Le rapport $U$ entre les intensités du courant de pompage de charge, $I_{\mathrm{CP} 1}$ et $I_{\mathrm{CP} 2}$ avant et après contrainte est donné par:

$$
U=\frac{I_{\mathrm{CP} 2}}{I_{\mathrm{CP} 1}}=\frac{K_{2} \cdot \int_{-L_{\mathrm{eff}} / 2}^{L_{\mathrm{eff}} / 2} N_{\mathrm{it} 2}(x) \mathrm{d} x}{K_{1} \cdot \int_{-L_{\mathrm{eff}} / 2}^{L_{\mathrm{eff}} / 2} N_{\mathrm{it} 1}(x) \mathrm{d} x} .
$$

En dérivant $U$ par rapport à $V_{\mathrm{R}}$, on obtient :

$$
\frac{\mathrm{d} U}{\mathrm{~d} V_{\mathrm{R}}}=\frac{\mathrm{d}\left(K_{2} / K_{1}\right)}{\mathrm{d} V_{\mathrm{R}}} \cdot \frac{K_{1}}{K_{2}} \cdot U+\frac{K_{2}}{K_{1}} \frac{\mathrm{d}}{\mathrm{d} V_{\mathrm{R}}}\left(\frac{\int_{-L_{\mathrm{eff}} / 2}^{L_{\mathrm{eff}} / 2} N_{\mathrm{it} 2}(x) \mathrm{d} x}{\int_{-L_{\text {eff }} / 2}^{L_{\mathrm{eff}} / 2} N_{\mathrm{it} 1}(x) \mathrm{d} x}\right) .
$$

En supposant $\left(N_{\mathrm{it} 1}\left(-L_{\mathrm{eff}} / 2\right)=N_{\mathrm{it} 1}\left(L_{\mathrm{eff}} / 2\right)=N_{\mathrm{it} 2}\left(-L_{\mathrm{eff}} / 2\right)\right)$, il vient :

$$
\frac{\mathrm{d} U}{\mathrm{~d} V_{\mathrm{R}}}=\frac{K_{1}}{K_{2}} \cdot U \cdot \frac{\mathrm{d}\left(K_{2} / K_{1}\right)}{\mathrm{d} V_{\mathrm{R}}}+\frac{K_{2}}{2 K_{1}} \frac{\mathrm{d} L_{\mathrm{eff}}}{\mathrm{d} V_{\mathrm{R}}} \cdot\left\{\frac{\frac{I_{\mathrm{CP} 1}}{K_{1}}\left[N_{\mathrm{it} 2}\left(L_{\mathrm{eff}} / 2\right)+N_{\mathrm{it} 1}\left(L_{\mathrm{eff}} / 2\right)\right]-\frac{2 I_{\mathrm{CP} 2}}{K_{2}} \cdot N_{\mathrm{it} 1}\left(L_{\mathrm{eff}} / 2\right)}{\left(I_{\mathrm{CP} 1} / K_{1}\right)^{2}}\right\}
$$

Soit,

$$
\frac{\mathrm{d} U}{\mathrm{~d} V_{\mathrm{R}}}=\frac{K_{1}}{K_{2}} U \frac{\mathrm{d}\left(K_{2} / K_{1}\right)}{\mathrm{d} V_{\mathrm{R}}}+K_{2} \frac{\mathrm{d} L_{\mathrm{eff}} / \mathrm{d} V_{\mathrm{R}}}{2 I_{\mathrm{CP} 1}} \cdot\left[N_{\mathrm{it} 2}\left(L_{\mathrm{eff}} / 2\right)+\left(1-\frac{2 K_{1}}{K_{2}} \cdot U\right) N_{\mathrm{it} 1}\left(L_{\mathrm{eff}} / 2\right)\right]
$$


$\mathrm{d} L_{\text {eff }} / \mathrm{d} V_{\mathrm{R}}$ peut être calculé en utilisant les équations (4) et (5) :

$$
\frac{\mathrm{d} L_{\mathrm{eff}}}{\mathrm{d} V_{\mathrm{R}}}=-\left(\frac{2 \varepsilon_{\mathrm{Si}}}{q N_{\mathrm{A}}}\right)^{1 / 2} \cdot\left(V_{\mathrm{R}}+2 \Psi_{\mathrm{B}}\right)^{-1 / 2} .
$$

Pour le calcul de $\mathrm{d}\left(K_{2} / K_{1}\right) / \mathrm{d} L_{\text {eff }}$, on note :

$$
\begin{gathered}
C=\frac{v_{\mathrm{th}} n_{\mathrm{i}} \sqrt{t_{\mathrm{r}}} t_{\mathrm{f}}}{\left|\Delta V_{\mathrm{G}}\right|} \\
\boldsymbol{\sigma}_{\mathrm{m} 1}=\sqrt{\boldsymbol{\sigma}_{\mathrm{n} 1}} \boldsymbol{\sigma}_{\mathrm{p} 1}, \quad \boldsymbol{\sigma}_{\mathrm{m} 2}=\sqrt{\boldsymbol{\sigma}_{\mathrm{n} 2}} \boldsymbol{\sigma}_{\mathrm{p} 2}
\end{gathered}
$$

et d'après (6),

$$
\left|V_{\mathrm{FB} 1}-V_{\mathrm{T} 1}\right|=\left|V_{\mathrm{FB} 2}-V_{\mathrm{T} 2}\right|=\left|V_{\mathrm{FB}}-V_{\mathrm{T}}\right|
$$

$\sigma_{\mathrm{m} 1}$ et $\sigma_{\mathrm{m} 2}$ sont les sections efficaces de capture moyennes des états d'interface avant et après dégradation. Elles sont obtenues par la méthode décrite au paragraphe 2.2.

$\mathrm{d}\left(K_{2} / K_{1}\right) / \mathrm{d} V_{\mathrm{R}}$ est alors donné par :

$$
\frac{\mathrm{d}\left(K_{2} / K_{1}\right)}{\mathrm{d} V_{\mathrm{R}}}=\frac{\ln \left(\sigma_{\mathrm{m} 1} / \sigma_{\mathrm{m} 2}\right)}{\left[\ln \left(C \sigma_{\mathrm{m} 1}\left|V_{\mathrm{FB}}-V_{\mathrm{T}}\right|\right)\right]^{2}} \cdot \frac{1}{\left|V_{\mathrm{FB}}-V_{\mathrm{T}}\right|} \cdot \frac{\mathrm{d}\left(\left|V_{\mathrm{FB}}-V_{\mathrm{T}}\right|\right)}{\mathrm{d} V_{\mathrm{R}}} .
$$

D'après l'équation (6), $\left|V_{\mathrm{FB}}-V_{\mathrm{T}}\right|$ peut s'écrire :

$$
\left|V_{\mathrm{FB}}-V_{\mathrm{T}}\right|=2 \Psi_{\mathrm{B}}+V_{\mathrm{R}}+\frac{\left\{2 q \varepsilon_{\mathrm{Si}} N_{\mathrm{A}}\left(V_{\mathrm{R}}+2 \Psi_{\mathrm{B}}\right)\right\}^{1 / 2}}{\varepsilon_{\mathrm{ox}} / e_{\mathrm{ox}}}+\Delta V_{\mathrm{T}} .
$$

En prenant la dérivée de $\left|V_{\mathrm{FB}}-V_{\mathrm{T}}\right|$ par rapport à $V_{\mathrm{R}}$, on obtient :

$$
\frac{\mathrm{d}\left(\left|V_{\mathrm{FB}}-V_{\mathrm{T}}\right|\right)}{\mathrm{d} V_{\mathrm{R}}}=1+\frac{\left(2 q \varepsilon_{\mathrm{Si}} N_{\mathrm{A}}\right)^{1 / 2}}{2 \varepsilon_{\mathrm{ox}} / e_{\mathrm{ox}}} \cdot\left(V_{\mathrm{R}}+2 \Psi_{\mathrm{B}}\right)^{-1 / 2}+\frac{\mathrm{d} \Delta V_{\mathrm{T}}}{\mathrm{d} V_{\mathrm{R}}}
$$

$\mathrm{d} \Delta V_{\mathrm{T}} / \mathrm{d} V_{\mathrm{R}}$ pouvant être calculé à partir des équations (4) et (7), $\mathrm{d}\left(\left|V_{\mathrm{FB}}-V_{\mathrm{T}}\right|\right) / \mathrm{d} V_{\mathrm{R}}$ devient :

$$
\begin{aligned}
\frac{\mathrm{d}\left(\left|V_{\mathrm{FB}}-V_{\mathrm{T}}\right|\right)}{\mathrm{d} V_{\mathrm{R}}}=1+\frac{\left(2 q \varepsilon_{\mathrm{Si}} N_{\mathrm{A}}\right)^{1 / 2}}{2 \varepsilon_{\mathrm{ox}} / e_{\mathrm{ox}}}( & \left.V_{\mathrm{R}}+2 \Psi_{\mathrm{B}}\right)^{-1 / 2} \times \\
& \times\left\{1-\frac{W_{\mathrm{m}}}{L}\left(1+\frac{2 W_{\mathrm{m}}}{r_{\mathrm{j}}}\right)^{-1 / 2}+\frac{r_{\mathrm{j}}}{L}\left[1-\left(1+\frac{2 W_{\mathrm{m}}}{r_{\mathrm{j}}}\right)^{1 / 2}\right]\right\}
\end{aligned}
$$

En utilisant l'expression ci-dessus de $\mathrm{d}\left(\left|V_{\mathrm{FB}}-V_{\mathrm{T}}\right|\right) / \mathrm{d} V_{\mathrm{R}}, \mathrm{d}\left(K_{2} / K_{1}\right) / \mathrm{d} V_{\mathrm{R}}$ s'obtient à partir de l'équation (23). Finalement la densité des états d'interface près du drain est obtenue en utilisant l'équation (20):

$$
N_{\text {it } 2}\left(L_{\text {eff }} / 2\right)=\frac{2 I_{\mathrm{CP} 1}}{K_{2}\left(\mathrm{~d} L_{\text {eff }} / \mathrm{d} V_{\mathrm{R}}\right)}\left[\frac{\mathrm{d} U}{\mathrm{~d} V_{\mathrm{R}}}-\frac{K_{1}}{K_{2}} \cdot U \frac{\mathrm{d}\left(K_{2} / K_{1}\right)}{\mathrm{d} V_{\mathrm{R}}}\right]+\left(\frac{2 K_{1}}{K_{2}} U-1\right) N_{\text {it1 }}\left(L_{\text {eff }} / 2\right)
$$

où $\mathrm{d} L_{\text {eff }} / \mathrm{d} V_{\mathrm{R}}$ est donnée par l'équation (21).

\section{Bibliographie}

[1] Balland, B., Plossu, C., Bardy, S. et Pinard, P., Rev. Phys. Appl. 20 (1985) 225.

[2] Balland, B., Plossu, C. et BARdy, S., Thin Solids Films 148 (1987) 175.

[3] Ning, T. H., Osburn, C. M. et Yu, H. N., J. Electronic Mat. 6 (1977) 65.
[4] Balland, B., Instabilities in Silicon Devices, vol. 1, chap. 2 et 9, Eds. G. Barbottin, A. Vapaille (North-Holland) Amsterdam (1986), pp. 101 et 441.

[5] Van Overstraeten, R. J., Declerck, G., Muls, P. A., IEEE Trans. ED 22 (1975) 282. 
[6] Wang, K. L. et Evwaraye, A. O., J. Appl. Phys. 47 (1976) 4574.

[7] Klassen, F. M., IEEE Trans. ED-18 (1971) 887.

[8] Brugler, J. S. et Jespers, P. G. A., IEEE Trans. ED 16 (1969) 297.

[9] Elliot, B. M., Solid-state Electron 19 (1976) 241.

[10] OwCZareK, A. et Kolodziejski, J. F., Electron Technol. 10 (1977) 55.

[11] Groeseneken, G., Maes, H. E., Beltran, N. et DeKeERSMAeCKer, R. F., IEEE Trans. ED-31 (1984) 42.

[12] Hofmann, K. R., Verner, C., Weber, W. et DORDA, G., IEEE Trans. Electron. Devices ED32 (1985) 691.

[13] Heremans, P., Maes, H. E. et Saks, N., IEEE Electron. Devices Lett. EDL-7 (1986) 428.
[14] Haddara, H. et Cristoloveanu, S., Solid State Electron. 29 (1986) 767.

[15] FoJT, R. et EISELE, I., Int. Conf. INFOS 87, Leuven, Belgium (Avril 1987).

[16] Maes, H. E. et Groeseneken, G., Electron. Lett. 18 (1982) 372.

[17] SzE, S. M., Physics of Semiconductor Devices (Wiley, Interscience) New-York (1981), pp. 431486.

[18] Hartmann, J. et Martin, F., Le vide et les Couches Minces, Suppl. au $N^{\circ} 218$ (1983).

[19] Declerck, M., Jespers, P., Rev. HF. Acta Tech. Belgica 9 (1974) 244.

[20] Sanders, F. H. M., Dieleman, J., Le vide et les Couches Minces, Suppl. au N 229 (1985). 